\section{A Diagnostic Conundrum: Acute Intermittent Porphyria Coexisting with Lumbar Prolapsed Intervertebral Disc}

Sir,

Abdominal pain accounts for $5-10 \%$ of emergency department consultations; this can be due to a wide range of pathologies. ${ }^{1}$ Few life-threatening conditions although uncommon, are worth considering - abdominal aortic aneurysm, mesenteric ischaemia, perforation of gastrointestinal tract, acute bowel obstruction, volvulus, ectopic pregnancy, placental abruption, myocardial infarction, and splenic rupture. ${ }^{1}$ Occasionally, conditions affecting the lower back may lead to diagnostic confusion because of associated or referred abdominal pain.

Acute intermittent porphyria (AIP) is a rare autosomal dominant inborn error of metabolism of the haem biosynthetic pathway, which can be exacerbated through a multitude of environmental factors, e.g. medicines, alcohol, hormonal changes, fasting or stress.2,3 AIP clinically manifests with severe abdominal pain, confusion, and seizures, which at times may be life-threatening. ${ }^{2}$ Other symptoms of AIP such as anxiety, depression, weakness, nausea, vomiting, constipation, hypertension and palpitation can mimic less serious pathologies and may cause a diagnostic conundrum.2,3 Pain in the chest, back, or lower extremities are also known to be the initial clinical manifestations of AIP. 3

A previously healthy 20 -year girl presented with history of acute abdominal pain, an episode of diarrhoea and recurrent episodes of vomiting. Clinical examination and observations of the vital signs, including blood pressure, were essentially normal. Urinalysis was reported as no abnormality detected. A provisional diagnosis of gastroenteritis was made and she was admitted for rehydration and symptomatic management with intravenous fluids, omeprazole and ondansetron. Evaluation by the gastroenterologist, the following day, did not identify any serious pathology; and hence she was discharged on symptomatic treatment.

She apparently did well for next two to three days, but then needed to be readmitted again as she developed severe low backache severely limitating her mobility. Neurosurgical review was requested and an MRI scan of the spine revealed lumbar prolapsed intervertebral disc (PIVD) with large L5 to S1 extruded disc. She was started on pain management with good response, initially. Strict bedrest and monitoring was advised.
The patient again developed acute abdominal pain and urinary tract infection - like symptoms with normal urinalysis three days later, while still being an inpatient. Pain in the abdomen was poorly localised, associated with few intermittent episodes of nausea and vomiting: and she had developed other new symptoms, such as anxiety and depression with occasional irritability and restlessness. All basic investigations, including CT scan of the abdomen, remained inconclusive. Psychiatry opinion was also requested.

However, next day, the urine dipstick revealed a large number of RBCs and the patient appeared very pale with persistence of symptoms. Repeat full blood count revealed a drastic fall in haemoglobin level from 11.7 $\mathrm{gm} / \mathrm{dL}$ at admission to $<7 \mathrm{gm} / \mathrm{dL}$. In view of her symptoms, which remained unexplained thus far, urinary porphobilinogen was requested. This was found to be positive (9.8 $\mathrm{mg} / \mathrm{L}$, normal range $0-4 \mathrm{mg} / \mathrm{L})$. In view of the elevated level of porphobilinogen on a spot urine test during the acute attack, a diagnosis of AIP was eventually established. She was kept nil by mouth, given intravenous dextrose, opoid analgesics, omeprazole and ondansetron. The patient was treated with human haemin infusion [dose $3 \mathrm{mg} / \mathrm{kg} /$ dose once daily] for 4 days. ${ }^{4}$ Her symptoms improved and she was discharged home after an inpatient stay of 10 days from the initial admission.

The patient was advised to continue her follow-up with the haematologist as few patients are known to develop recurrent attacks. This was also to ensure that she is monitored for late complications which include chronic pain, hepatocellular carcinoma, chronic renal failure and hypertension. ${ }^{4}$ She was provided with a list of medications to be avoided.

Human haematin is often not readily available in resource-limited settings, other therapeutic options such as haemodialysis may be considered as there is some evidence in the acute setting for AIP being successfully treated with dialytic support or as a transient measure till human haematin can be procured/imported. 5

Although, in retrospect, the patient had all the classical symptoms of AIP, the diagnosis got established only after a considerable delay of 8 days from initial presentation. This was partly due to vagueness of her initial symptoms, which got misinterpreted as gastroenteritis initially and largely due to the co-incidental findings of lumbar PIVD. It is important that a balance is maintained where there may co-exist two different pathologies. A recently published case of AIP in a 42-year woman from the UK highlighted similar challenges with a delay in diagnosis as too many key aspects of her history were initially not known, and others got ignored or misinterpreted.2 
Seizures are known to occur in $20-30 \%$ cases during acute attacks of AIP. 3 The most commonly reported electrolyte abnormality is hyponatremia. Moreover, hypomagnesemia is also common. ${ }^{3}$ Both are risk factors for development of seizures, hence regular monitoring of electrolytes is suggested during acute attacks of AIP. 3 Symptoms of AIP patients may change during the menstrual cycles as reported by a Swedish study where $30 / 32$ women showed significant cyclicity in at least one AIP or premenstrual syndrome (PMS) symptoms. ${ }^{6}$ Back pain and sweet craving were the most frequent AIP pain and PMS symptoms, respectively. ${ }^{6}$ Pelvic pain and abdominal swelling showed the strongest correlation. 6

It is essential that the diagnosis of AIP is considered and appropriate investigations are initiated in a time-critical manner. Early treatment is likely to prevent significant morbidity and mortality. The case study also reflects the unexpected diagnostic challenges that may be faced by specialist clinicians in a patient presenting with two different pathologies simultaneously. Adequate knowledge about AIP is, therefore, essential for clinicians from all specialities as has been vividly demonstrated here as the management of this patient at the time of diagnosis of AIP was being led by the neurosurgical team.

\section{CONFLICT OF INTEREST:}

Authors declared no conflict of interest.

\section{AUTHORS' CONTRIBUTION:}

SA: Concept, data collection, manuscript editing and revision, and provided expert neurosurgical opinion.
SPP: Manuscript preparation and revision; literature search, submission and correspondence.

\section{REFERENCES}

1. Macaluso CR, McNamara RM. Evaluation and management of acute abdominal pain in the emergency department. Int $J$ Gen Med 2012; 5:789-97.

2. O'Malley R, Rao G, Stein P, Bandmann O. Porphyria: Often discussed but too often missed. Pract Neurol 2018; 18:352-8.

3. Besur S, Schmeltzer P, Bonkovsky HL. Acute porphyrias. J Emerg Med 2015; 49:305-12.

4. Stein PE, Badminton MN, Rees DC. Update review of the acute porphyrias. Br J Haematol 2017; 176:527-8.

5. Prabahar MR, Manorajan R, Sathiyakumar D, Soundararajan $P$, Jayakumar M. Hemodialysis: A therapeutic option for severe attacks of acute intermittent porphyria in developing countries. Hemodial Int 2008; 12:34-8.

6. Ahangari A, Bäckström T, Innala E, Andersson C, Turkmen S. Acute intermittent porphyria symptoms during the menstrual cycle. Intern Med J 2015; 45:725-31.

Shameem Ahmed ${ }^{1}$ and Siba Prosad Paul ${ }^{2}$

1 Department of Neurosurgery................................. Hospitals, (Unit: International Hospital), Guwahati, India.

2 Department of Paediatrics, Torbay Hospital, Torquay, UK Correspondence to: Dr Siba Prosad Paul, Department of Paediatrics, Torbay Hospital, Vowden Hall, Lowes Bridge, Torquay, TQ2 7AA, UK

E-mail:siba@doctors.org.uk

Received: January 14, 2019; Revised: April 01, 2019; Accepted: April 06, 2019 